\title{
Radiological findings in relation to the neurodevelopmental outcome in hydrocephalic children treated with shunt insertion or endoscopic third ventriculostomy
}

\author{
Patrycja Larysz $\cdot$ Dawid Larysz $•$ Marek Mandera
}

Received: 10 January 2013 / Accepted: 5 June 2013 / Published online: 22 June 2013

(C) The Author(s) 2013. This article is published with open access at Springerlink.com

\begin{abstract}
Purpose The aim of the study was to compare the radiological indicators of effectiveness for hydrocephalus treatment in children operated on under the third year of age with the use of shunt insertion (SI) and endoscopic third ventriculostomy (ETV). The effectiveness was considered in terms of postoperative neurodevelopment in correlation to pre- and postoperative radiological findings.

Methods The examined group consisted of 46 children operated on for hydrocephalus in the Division of Pediatric Neurosurgery in Katowice, Poland. There were 21 children treated with SI and 25 with ETV. The radiographic assessment was carried out on the basis of MRI and CT examinations with the use of a linear estimate known as frontal and occipital horn ratio (FOR). The FOR values were calculated for the entire group and in correlation to the treatment method and to the children neurodevelopment evaluated with The Denver Developmental Screening Test.

Results No differences were recognized between initial FOR value in terms of the postoperative children neurodevelopment. In the successful ETV-treated subgroup, the mean change in FOR was 0.05 and in the SI-treated subgroup, the mean change in FOR 0.13 . The patients with BFOR $>0.1$, developed normally more often than those in whom BFOR was lower than 0.1. Conclusions The initial FOR value probably does not affect the postoperative developmental outcome. Long-term change in ventricles size after surgery can correlate with psychomotor development of hydrocephalic children. Presumably, there are no differences between two treatment options according to initial FOR values and to changes in FOR values.
\end{abstract}

Keywords Children $\cdot$ Hydrocephalus $\cdot$ Development . Neuroradiology

P. Larysz $(\bowtie) \cdot$ D. Larysz $\cdot$ M. Mandera

Division of Pediatric Neurosurgery, Medical University of Silesia,

Upper-Silesian Children's Health Centre, Katowice, Poland

e-mail: lapat@poczta.onet.pl

\section{Introduction}

Hydrocephalus is most commonly treated with shunt insertion (SI) and/or endoscopic third ventriculostomy (ETV). In spite of the many attempts at comparing the surgery methods in question, there has been no definite answer as to which method of treating hydrocephalus in a population of young children is superior and which determinants to use to evaluate effectiveness in young patients [8]. Many studies tend to neglect the crucial aspect, which is child development $[2,5$, $14,15]$. To consider treatment effectiveness, not only symptoms of active hydrocephalus should be resolved but also the best possible conditions for further development and the best possible quality of life for the child be ensured [1, 13, 15, 21]. The influential mechanisms of hydrocephalus on a developing brain have not yet been researched, which makes prognosis of the psychomotor development more difficult. Endoscopic third ventriculostomy, as the method which restores a more "physiologic" cerebrospinal fluid (CSF) flow, does not always result in the expected improvement of psychomotor development [3,11, 17]. Shunts, in turn, in spite of ensuring effective CSF drainage, bring about a number of possible complications deteriorating the psychomotor status, as for example seizures and neuroinfection [4, $8,18,19]$. One of the most crucial criteria for the outcome evaluation seems to be efficient CSF drainage assessed on the basis of a clinical examination and, secondly, of a radiological examination. The pre- and postoperative measurement of the ventricular size could take part in the prediction of developmental outcome of hydrocephalic child. The most accurate measurement based on ultrasonography (USG), computed tomography (CT), and/or magnetic resonance imaging (MRI) examinations at present are the volumetric measurements. O'Hayon et al. stress the role of the frontal and occipital horn ratio (FOR), originated by them in 1998, as a simple, effective, and credible linear method of measuring the width of the ventricles, possible to conduct with the 
aid of all imaging examinations-USG, CT, and MRI. FOR has been proved to have the supreme linear correlation to the measurement of the ventricular volume $(R=0.852)$ among nonvolumetric measurements. The authors believe therefore, that it is one of the indicators that should be used in practice $[7,12,23]$.

Aim

The aim of the study was to compare the radiological indicators (FOR) of effectiveness for hydrocephalus treatment in children operated on under the third year of age with the use of shunt insertion and endoscopic third ventriculostomy. Effectiveness was considered in terms of postoperative psychomotor development in correlation to pre- and postoperative radiological findings.

\section{Patients}

General description The examined group consisted of 46 children operated on for hydrocephalus at the Division of Pediatric Neurosurgery at the Upper-Silesian Children's Health Centre in Katowice. The group included 23 girls and 23 boys. The follow-up for the children examined was between 7 and 112 months (mean, 36.7 months; median, 28 months). Hydrocephalus symptoms were manifested between birth up to 32 months, with a median of 1 month. The age of the examined children at the time of study inclusion ranged from 1 up to 33 months (mean, 5.6 months; median, 2.0 months). Hence, all the patients were under 3 years of age at the time of surgery. In 28 children, hydrocephalus was congenital (61\%)-presented at birth and caused either by events or influences that occurred during fetal development (no evidence of inflammation or hemorrhage were observed) or genetic abnormalities; communicating or noncommunicating, including aqueductal stenosis. In eight cases (17\%), postinflammatory hydrocephalus was recognized; whereas in 10 patients (39\%), hydrocephalus was caused by an intracranial hemorrhage.

Children treated with shunt insertion The group consisted of 21 children, making up $45.6 \%$ of the analyzed group, including 10 girls and 11 boys. The children's age at the time of the first surgery was between 1 and 14 months (median, 2.0 mean, 2.8 months). The etiology analysis of hydrocephalus revealed that six children $(28.6 \%)$ displayed congenital hydrocephalus, another six post-inflammatory $(28.6 \%)$, whereas nine cases resulted from an intracranial hemorrhage.

Children treated with endoscopic third ventriculostomy The group consisted of 25 children, making up for $54.3 \%$ of the analyzed group, including 13 girls and 12 boys. The children's age at the time of the first surgery ranged from 1 to
33 months (median, 4.0 months; mean, 7.9 months). The analysis of the etiology of the hydrocephalus revealed that it was congenital in 22 patients, was post-inflammatory $(8.0 \%)$ in two, whereas one case $(4 \%)$ resulted from an intracranial hemorrhage.

\section{Methods}

The radiographic assessment was carried out on the basis of MRI and CT examinations with the use of a linear estimate known as FOR. FOR is the sum of the widths of frontal and occipital horns of the ventricular system divided by a double width of the parietal lobes in a cross-section view. The normal frontal and occipital ratio equals $0.37(99 \% \mathrm{CI}, 0.36-0.38)$ and is independent of age [16]. We used the term "normal ventricles" with regard to normal-sized ventricles $(\mathrm{FOR}<0.37)$ and without any other severe abnormalities, as for example tumor, Dandy-Walker syndrome, myelomeningocele, etc. First, we attempted to determine the frequency of size normalization of the ventricular system in correlation to the method of treatment and to the age when the first hydrocephalus symptoms were manifested. We also investigated the influence of age at the time of the first surgery and of the etiology of hydrocephalus on the likeliness of restoring normal ventricular width. Then, we investigated whether ventricular size normalization determined normal development. The values of the attained FOR were analyzed in correlation to the method of treatment. Mean FOR values were calculated along with mean changes in FOR values for the entire group and in correlation to the treatment method. Subsequently, we tried to determine whether the initial FOR value influenced normal development. Therefore, the patients were divided into two subgroups depending on the initial FOR value in the first examination, with a cutoff value set at 0.6 (for the whole group, the mean initial FOR value was 0.59 ; median, 0.60$)$. Next, the change in FOR value $(\Delta F O R)$ was analyzed. $\triangle \mathrm{FOR}$ was calculated using the following

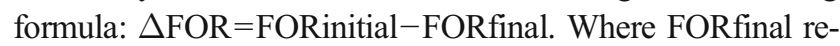
fers to the estimate calculated on the basis of the last available neuroimaging examination in the child's wellbeing, whereas FORinitial is the initial value of the estimate in the examination before treatment was started. In the next stage, hoping to determine the effect changes FOR have on normal development and on the effectiveness of treatment, we divided the children into two subgroups depending on the change in FOR with cutoff values set at $\Delta \mathrm{FOR}=0.1$ and 0.025 .

We assessed the success/failure ratio for both methods - ETV and shunting. The efficient CSF drainage, which means without any symptoms of intracranial hypertension assessed on the basis of a clinical examination and radiological examination, was considered as the success of the treatment. 
The evaluation of psychomotor development of the children treated for hydrocephalus was carried out with the Denver Developmental Screening Test. The examination reveals delays in reaching certain milestones and abilities, as well as developmental disharmony and therefore, evaluated whether development was normal, or not, without determining the characteristics of the disorders. The evaluation of the psychomotor development of the patients was conducted after a minimum of 6 months from surgery. Subject to evaluation were the presence of harmonious development and the following developmental modalities: motor skill, posture control, language, visual-motor coordination, and personalsocial sphere. The child's development was assessed as "normal" provided it was harmonious and within the norm for each of the developmental modalities.

Statistical analysis was performed using standard statistical software (Statistica v8.0 StatSoft). The significance level was set at $p<0.5$. All variables were tested for normality using the Kolmogorov-Smirnov and the Shapiro-Wilk tests. When the test statistic followed a normal distribution and the tested populations had the same variance (testable using Snedecor's test), Student's $t$ test was used. In a situation when distributions of variables did not show a normal distribution, or the samples taken were so small that it was impossible to tell if they were part of a normal distribution or not, the Mann-Whitney $U$ test was used. The chi-square test of independence was used to assess whether paired observations on two variables, expressed in a contingency table, were independent of each other. For smaller data, Yates' correction for continuity was used. Fisher's exact test was used in the analysis of $2 \times 2$ contingency tables. Alternatively, to calculate greater tables, the maximum likelihood estimates were used.

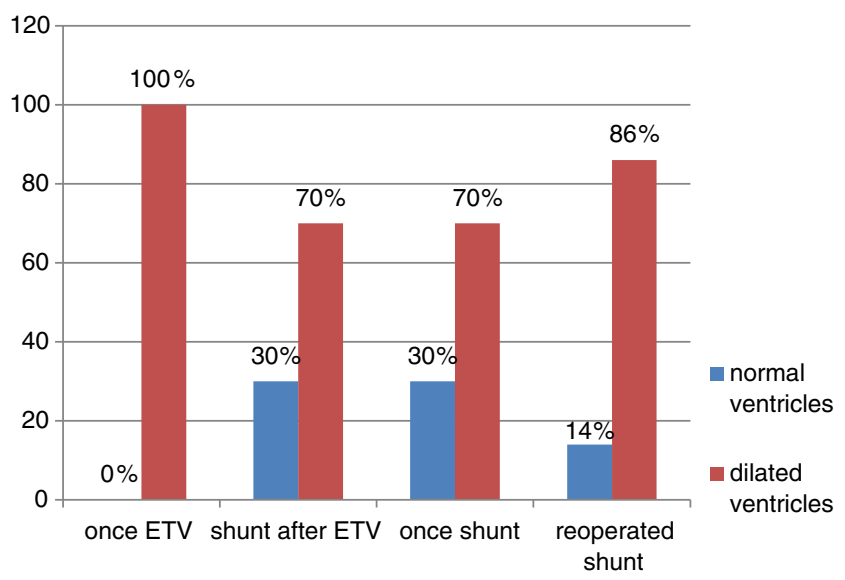

Fig. 1 Normalization of ventricular size according to treatment. The ventricles width remained dilated in all children treated clinically successful with ETV. Normal ventricular size was observed in $30 \%$ of children unsuccessfully treated with ETV and subsequently with SI and in the group operated successfully with the first SI
The study has been approved by the appropriate ethics committee. All persons/parents gave their informed consent prior to their inclusion in the study.

\section{Results}

Normalization of ventricular size In follow-up imaging examinations after surgery, the normalization of the ventricles size was revealed in $19.51 \%$ of the patients. In children originally operated by means of ETV, the prevalence was $14.29 \%$ whereas in children treated originally with SI, it equaled $25.00 \%(p=0.003)$. The success rate of ETV was $60 \%$ and after shunting was $66.6 \%$. All children treated unsuccessfully with ETV underwent shunt insertion. In all patients successfully treated clinically with ETV, the width of the ventricles remained abnormal, whereas in the group of children unsuccessfully treated with ETV and subsequently with SI (10 children), the proportion of children with a normal ventricular width revealed in follow-up examinations equaled $30.00 \%$. In the children who were operated once with the SI method, the frequency of recovered normal ventricular width equaled $30.79 \%$ and in the reoperated children (seven children; Fig. 1) equaled $14.28 \%$.

Normalization of ventricular size according to psychomotor development Next, it was investigated whether the presence in the neuroimaging examinations of recovered normal ventricular width correlated with the neurodevelopmental outcome. Less than $10 \%$ of children whose ventricular system remained dilated were found to develop normally, in spite of lacking symptoms of active intracranial pressure. Half of the studied group with postoperative normal ventricular width developed abnormally (Fig. 2). The difference was statistically significant $(p=0.045)$.

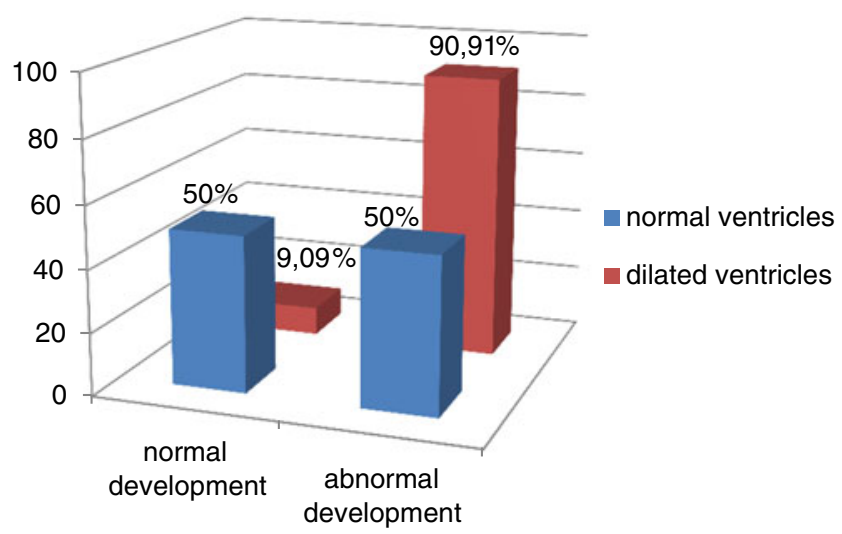

Fig. 2 Child development in correlation to the normalization of ventricular size. Over $90 \%$ of children whose ventricles remained dilated were found to develop abnormally. Despite postoperative normalization of ventricular size, $50 \%$ of children developed abnormally 
Normalization of ventricular size according to the age of the hydrocephalus manifestation, to the age at the time of the first surgery, and to the etiology of hydrocephalus The ventricular system was restored to its normal width upon treatment significantly more often in patients who were diagnosed older than in the first month of age (23.53 vs. $19.05 \%, p=0.0088$ ); nonetheless, in the majority of children ( 80.95 vs. $76.47 \%$, respectively), the ventricular system did not recover to its normal volume. A similar, statistically significant correlation was observed dividing children into subgroups, where the cutoff value was 6 months of age ( $20 \%$, below 6 months; $25 \%$, over 6 months). Investigation into the influence of age at the time of the first surgery and of the etiology of hydrocephalus on the likeliness of restoring normal ventricular width revealed no statistically significant difference between the examined subgroups.

The analysis of FOR values The preoperative FOR values were analyzed. For the whole group, the mean FOR value was 0.59 (median, 0.60 ; range, $0.38-0.83$ ). For SI, the mean initial FOR value was 0.6 (median, 0.64 ; range, $0.38-0.68$ ), whereas for ETV the mean initial value was 0.59 (median, 0.58 , range, $0.40-0.83$ ). No statistically significant difference was found between these groups.

Next, the group of examined children was divided into two subgroups due to the FOR value in the preoperative examination. The cutoff value was set at 0.6 . No statistically significant differences were recognized in the subgroups in terms of child development. In the group with FOR value below $0.6,18.8 \%$ of children developed normally, whereas in the group with FOR value over 0.6, normal development was observed in $16.67 \%$ of children.

A mean change in FOR in the entire group was 0.08 (median, 0.01; range, 0.05-0.036). It was 0.05 (median,

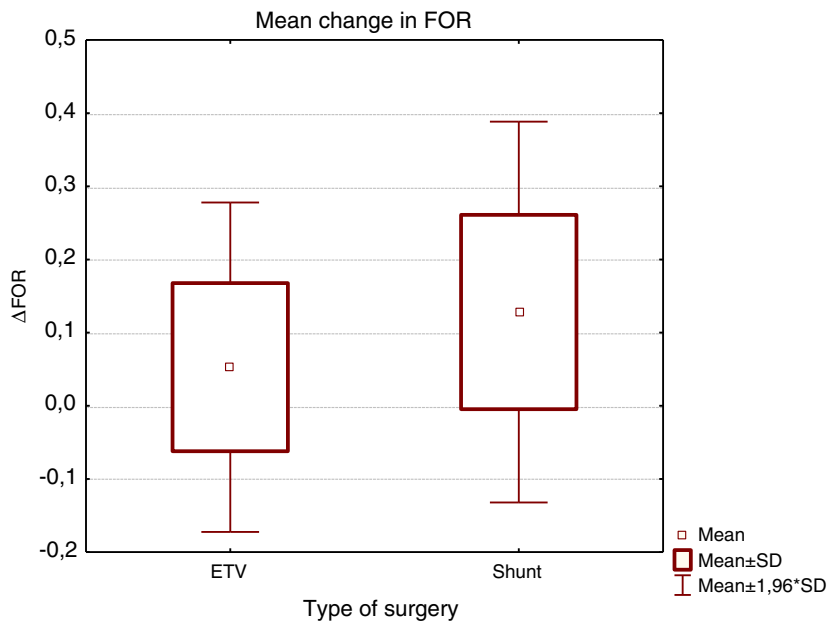

Fig. 3 Mean change in the FOR estimated in the analyzed SI and ETV subgroups. A tendency towards greater change was observed after SI when comparing FOR changes $(p<0.05)$

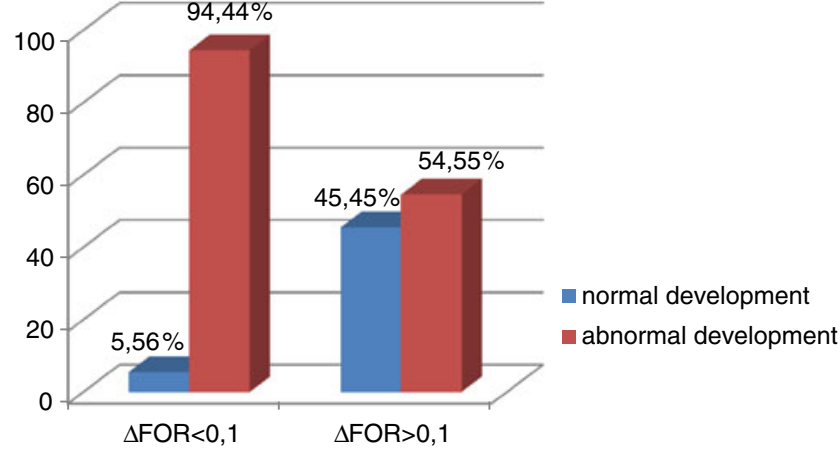

Fig. 4 Children development following surgery in correlation to $\triangle F O R$

0.005; range, $0.05-0.34)$ in the successfully ETV-treated subgroup and 0.13 (median, 0.13; range, 0.05-0.36) in the SI-treated subgroup. The comparison of FOR changes in the subgroups revealed no statistically significant difference. However, a tendency towards greater change was observed in the shunted group (Fig. 3).

Children development in correlation to the change in $\triangle F O R$ The children were divided into two subgroups according to the change in FOR value. The cutoff value was set at $\Delta \mathrm{FOR}=0.1$.

It was revealed that patients with $\triangle \mathrm{FOR}>0.1$, developed normally more often than those in whom $\triangle \mathrm{FOR}$ was lower than $0.1(p=0.0079)$. Detailed values are presented in Fig. 4 . A similar correlation was observed dividing children into subgroups, where the cutoff value was set at $\Delta \mathrm{FOR}=0.025$.

\section{Discussion}

The pre-operative and postoperative measurement of the ventricular width is an essential part of the diagnostics and the assessment of the effectiveness of hydrocephalus treatment. The change of the ventricular width after ETV often times does not correlate with the value of intracranial pressure and with the improvement of the clinical condition. Particularly, a lack of decrease in the ventricular size following ETV in children when improvement of the clinical condition occurs is, at present, not considered a failure in the treatment of hydrocephalus [6]. It may, however, suggest insufficient CSF absorption in these patients, with a likely prospect of postoperative development of a compensated communicating hydrocephalus. Such patients should remain under permanent control of neurologists and neuropsychologists. The observation of changes in the volume of the ventricles following successful ETV treatment revealed the most intensive diminishing of the ventricles to take place within 3-6 months after the surgery. Within the following months, stabilization occurs and the ventricles further decrease insignificantly or 
not at all - as opposed to the post-SI image, where the ventricles keep decreasing in size after a 6-month period, stabilizing around 12 months after the surgery. In some cases, after the initial period of size reduction, the ventricles were observed to increase slightly after around 2 years of surgery [7].

In the course of follow-up imaging examinations, we found that in $19.51 \%$ of the patients, the width of the ventricular system recovered to normal values. In children originally operated with the use of ETV, it was established that the ventricular system significantly less frequently recovered to normal values as compared to the children treated primarily with SI. Yet, in the SI-treated group, due to an unsuccessful ETV, the percentage of patients with normal ventricular width in the follow-up examinations equaled $30 \%$. Nonetheless, a normal ventricular size did not determine normal development. Namely, in half of the cases with normal ventricular size, abnormal development was still observed. In turn, the frequency of the normalization in ventricles size was twice as high in the children treated with SI once, as compared to those who were reoperated. The age at the time of the surgery and the etiology of the hydrocephalus did not affect the frequency of the normal size ventricles. As opposed to the results mentioned above, in our study, the children whose ventricles width normalized, revealed normal psychomotor development significantly more frequently.

Kulkarni et al. performed a retrospective comparison of postoperative radiological image of children with a normally and abnormally functioning ventriculocysternostomy, revealing a statistically significant difference in postoperative reduction in the ventricular size between the two groups. Using FOR estimate, they found a mean reduction of the size of the ventricles by $15 \%$ in the group with effective ETV and by $7 \%$ in the group with ineffective ETV [12]. Another investigation, using volumetric measurements, revealed a significant reduction in the volume of the lateral ventricles on average by $30 \%$ (from 5 to $80 \%$ ) within 3 weeks after a successfully performed ETV [20]. As opposed to ETV, following SI, the size of the cerebral ventricles decreases significantly and stabilizes on average between 6 and 12 months after first-time surgery [24]. All the same, the size of the ventricles rarely goes back to normal in SI-treated patients, similarly as in those treated with ETV. Moreover, as some authors report, the size of the ventricles poorly correlates with a long-term assessment of the children's psychomotor development [10]. In 2009, Warf et al. compared the size of the ventricular system with the use of FOR in children with hydrocephalus and myelomeningocele. They found no difference between the group treated with ETV, with SI and the group not requiring treatment due to ventriculomegaly. Moreover, these authors did not find any correlation between the size of the ventricles and the psychomotor outcome of the studied children. Yet, they revealed significantly more advanced development in the children with ventriculomegaly as compared to the children treated for active hydrocephalus, even though the examined groups displayed the same width of the ventricular system [23]. Another study, in turn, which had as its goal the assessment of outcome of 129 children with the use of SI, found a significant correlation between the initial dilatation of the ventricular system and the child's development. No correlation, however, was determined between the size of the ventricular system after the treatment and the final IQ, with the exception of cases where the dilatation remained considerable in spite of the treatment, where the IQ results were low [9].

While comparing the mean FOR values in our study, no significant difference was found between the patients treated originally with ETV and SI. Moreover, the initial FOR value before surgery turned out to be inconsequential in terms of further child development. In the group with preoperative FOR value below $0.6,18.8 \%$ of children developed normally, whereas in the group with FOR value over 0.6, normal development was observed in $16.67 \%$ of children. In turn, children with a larger change in FOR $(\Delta \mathrm{FOR}>0.025$ and $>0.1)$ in the final neurodevelopmental examination significantly developed normally more often than those in whom the $\Delta \mathrm{FOR}$ was lower (Fig. 4). The obtained results prove that the ventricular width before surgery has probably no effect on the postsurgical development of the patients. The change in FOR value following treatment indicates the likeliness of better future development of the patients. This remains consistent with the aforementioned reports where a significant decrease of the ventricular width in the cases of its more considerable dilatation before surgery was observed and where it was proved that the pretreatment ventricular width did not affect the children's postoperative development $[7,23]$.

In his study, Takahashi found that normal psychomotor development is most likely to take place in children whose brain image after surgery was normal in MRI examinations, which is confirmed by our study. When an abnormally developing CNS is revealed or/and changes secondary to hydrocephalus are found in MRI, in turn, SI may be required to obtain the optimum psychomotor development despite "clinically" normal control of intracranial pressure [22]. It has been also proved that the duration of untreated hydrocephalus negatively correlates with the extent of reduction in the ventricular size after surgery [7]. In our study, the ventricular system was restored to its normal width upon treatment significantly more often in patients who were diagnosed older than in the first month of age (23.53 vs. $19.05 \%, p=0.0088)$. However, the majority of children ( 80.95 vs. $76.47 \%$, respectively) presented postoperatively dilated ventricular system. A similar, statistically significant correlation was observed dividing children into subgroups, where the cutoff value was 6 months of age $(20 \%$, below 6 months of age; $25 \%$, over 6 months 
of age). We can suspect that the diagnosis of hydrocephalus in the first month of age probably refers to a pathology started during pregnancy. In these cases, the duration of untreated hydrocephalus was longer and we do not observe spectacular changes in the ventricular size after surgery.

\section{Conclusions}

The initial FOR value probably does not affect the postoperative developmental outcome. Long-term change in ventricles size after surgery can correlate with psychomotor development of hydrocephalic children. Presumably, there are no differences between two treatment options (ETV versus SI) according to initial FOR values and to changes in FOR values in young patients operated on for hydrocephalus. However, after shunt insertion, the probability of normalization of ventricles size is higher than after ETV and therefore the probability of normal development in shunted children should be higher.

Open Access This article is distributed under the terms of the Creative Commons Attribution License which permits any use, distribution, and reproduction in any medium, provided the original author(s) and the source are credited.

\section{References}

1. Beems T, Grotenhuis JA (2002) Is the success rate of endoscopic third ventriculostomy age-dependent? An analysis of the results of endoscopic third ventriculostomy in young children. Childs Nerv Syst 18:605-608

2. Bergsneider M, Egnor MR, Johnston M, Kranz D, Madsen JR, McAllister JP 2nd, Stewart C, Walker ML, Williams MA (2006) What we don't (but should) know about hydrocephalus. J Neurosurg 104(3 Suppl):157-9

3. Browd SR, Gottfried ON, Ragel BT, Kestle JRI (2006) Failure of cerebrospinal fluid shunts part II: overdrainage, loculation, and abdominal complications. Pediatr Neurol 34:171-176

4. Di Rocco C, Massimi L, Tamburrini G (2006) Shunts vs endoscopic third ventriculostomy in infants: are there different types and or rates of complications? A review. Childs Nerv Syst 22(12):1573-1589

5. Drake JM (2007) The surgical management of pediatric hydrocephalus. Neurosurgery 62(suppl 2):633-640

6. Garg AK, Suri A, Sharma BS, Shamim SA, Bal CS (2009) Changes in cerebral perfusion hormone profile and cerebrospinal fluid flow across the third ventriculostomy after endoscopic third ventriculostomy in patients with aqueductal stenosis: a prospective study. Neurosurg Pediatrics 3:29-36
7. George EST, Natarajan K, Sgouros S (2004) Changes in ventricular volume in hydrocephalic children following successful endoscopic third ventriculostomy. Childs Nerv Syst 20:834-838

8. Green AL, Pereira EA, Kelly D, Richards PG, Pike MG (2007) The changing face of paediatric hydrocephalus a decade's experience. J Clin Neurosci 14:1049-1054

9. Hoppe-Hirsch E, Laroussinie F, Brunet L, Sainte-Rose C, Renier D, Cinalli G, Zerah M, Pierre-Kahn A (1998) Late outcome of the surgical treatment of hydrocephalus. Child Nerv Syst 14:97-99

10. Kestle J, Drake J, Milner R, Sainte-Rose C, Cinalli G, Boop F, Piatt J, Haines S, Schiff S, Cochrane D, Steinbok P, MacNeil N (2000) Long-term follow-up data from the Shunt Design Trial. Pediatr Neurosurg 33(5):230-236

11. Li KW, Nelson C, Suk I, Jallo G (2005) Neuroendoscopy: past, present and future. Neurosurg Focus 19(6):E1

12. Kulkarni AV, Drake JM, Armstrong DC, Dirks PB (2000) Imaging correlates of successful endoscopic third ventriculostomy. J Neurosurg 92:915-919

13. Kulkarni AV, Drake JM, Kestle JRW, Mallucci CL, Sgouros S, Constantini S (2010) Endoscopic third ventriculostomy vs cerebrospinal fluid shunt in the treatment of hydrocephalus in children: a propensity score-adjusted analysis. Neurosurgery 67:588593

14. Kulkarni AV, Drake JM, Mallucci CL, Sgouros S, Roth J, Constantini S (2009) Endoscopic third ventriculostomy in the treatment of childhood hydrocephalus. J Pediatr 155:254-9

15. Kulkarni AV, Hui S, Shams I, Donnelly R (2010) Quality of life in obstructive hydrocephalus endoscopic third ventriculostomy compared to cerebrospinal fluid shunt. Childs Nerv Syst 26:7579

16. Kulkarni AV, Riva-Cambrin J, Browd SR (2011) Use of the ETV Success Score to explain the variation in reported endoscopic third ventriculostomy success rates among published case series of childhood hydrocephalus. J Neurosurg Pediatr $7(2): 143-6$

17. Li KW, Nelson C, Suk I, Jallo GI (2005) Neuroendoscopy: past, present and future. Neurosurg Focus 19(6):E1

18. O’Hayon BB, Drake JM, Ossip MG, Tuli S, Clarke M (1998) Frontal and occipital horn ratio: a linear estimate of ventricular size for multiple imaging modalities in pediatric hydrocephalus. Pediatr Neurosurg 29:245-249

19. Patwardhan RV, Nanda A (2005) Implanted ventricular shunts in the United States: the billion-dollar-a-year cost of hydrocephalus treatment. Neurosurgery 56:139-145

20. Schwartz TH, Ho B, Prestigiacomo CJ, Bruce JN, Feldstein NA, Goodman RR (1999) Ventricular volume following third ventriculostomy. J Neurosurg 91(1):20-5

21. Stachura K, Libionka W (2007) An outline of the history of neuroendoscopy. Medical review 64(2):118-120

22. Takahashi Y (2006) Long-term outcome and neurologic development after endoscopic third ventriculostomy versus shunting during infancy. Childs Nerv Syst 22:1591-1602

23. Warf B, Ondoma S, Kulkarni A, Donnelly R, Ampeire M, Akona J, Kabachelor CR, Mulondo R, Nsubuga BK (2009) Neurocognitive outcome and ventricular volume in children with myelomeningocele treated for hydrocephalus in Uganda. J Neurosurg Pediatrics 4(6):564-70

24. Xenos C, Sgouros S, Nataranjan K, Walsh AR, Hockley A (2003) Influence of shunt type on ventricular volume changes in children with hydrocephalus. J Neurosurg 98:277-283 